\title{
Виталий Огородников
}

Татьяна Архипова

Андрей Губанов

\section{ЭЛЕМЕНТЫ ПРОБЛЕМНОГО ОБУЧЕНИЯ ТЕОРИИ ПРЕДЕЛЬНЫХ СОСТОЯНИЙ В ЗАДАЧАХ СОПРОТИВЛЕНИЯ МАТЕРИАЛОВ}

Парадоксальность экономической ситуации в Украине заключается в том, что при избытке специалистов с высшим образованием экономика испытывает острую нехватку квалифицированных (компетентных) специалистов, способных выполнять определённые профессиональные и социальные функции. Компетентно-профессиональный подход в высшем образовании предполагает особую организацию педагогического процесса заведения высшего образования. Усиливается практический, межпредметный, прикладной аспекты образования. Это достигается не за счёт введения новых предметов или увеличения объёма дисциплин, а за счёт их содержательной переориентации от «декларативных» знаний (знать «что») к процедурным (знать «зачем и почему»).

В то время, как существующий квалификационный подход направлен на формирование у выпускника системы знаний, умений и навыков, обеспечивающих типичные виды профессиональной деятельности в стабильных условиях, компетентностный подход способствует формированию готовности к оптимальному решению профессиональных, социальных, личных проблем в быстро изменяющихся обстоятельствах информационного общества. Современных подходов требуют и учебные процессы изучения таких классических дисциплин, как химия, физика, сопротивление материалов и другие.

Студенты заведений высшего технического образования приступают к изучению дисциплины «Сопротивление материалов» после освоения курса теоретической механики. Кроме того, необходимы базовые знания физики и высшей математики.

Следовательно, обучаясь на инженерной специальности, нельзя обойти стороной дисциплину «Сопротивление материалов», поскольку сопромат является связующим звеном между фундаментальными дисциплинами общеинженерной подготовки, которые изучают в заведении высшего технического образования (ЗВТО) первые два года, и специальными, связанными с профессией будущего специалиста, поскольку, игнорируя фактор надёжности - предмет изучения сопромата, создание новой техники невозможно.

Несмотря на появление современных производительных компьютеров, прецизионных станков, роль изучения «сопротивления материалов» только возросла, поскольку создание новой техники определяется не только скоростью вычислений, но и, в большей степени, творческим потенциалом человека и знаниями, значительная доля которых должна принадлежать сопромату (Феодосьев, 2010; Dell, Y., Gese, H., Kessler, L., Werner, H., \& Hooputra, H., 2001).

\section{Зачем нужен сопромат}

Сопротивление материалов даёт представление о процессах, происходящих внутри материала конструкции при испытании им нагрузки, значительная часть которых в сопромате существует в виде гипотез и допущений. Однако это не мешает производить вычисления с инженерной точностью и прогнозировать, выдержит ли материал нагрузку во время эксплуатации. Понимание этих процессов определяет Ваш профессиональный уровень. Обладая знанием сопромата, Вы повысите эффективность Вашей работы, и если даже вы не будете знать, пригодятся ли Ваши наработки, Вы будете знать, для чего производите расчёт и станете уверенным в том, что конструкция не развалится в течение эксплуатационного периода.

\section{Сопромат и профессия инженера}

Сопромат, в отличие от фундаментальных дисциплин (математики, физики, теоретической механики и др.), требует в большей степени не умения использовать сложные расчёты, а творческий, инженерный подход к решению задачи сопротивления материалов, который заключается в умении выбирать достаточную, целесообразную точность, обеспечивающую сочетание надёжности конструкции, простоты технологии изготовления и экономичности. Если Вы обучаетесь на инженерной 
специальности, то должны понимать, что инженер должен не только уметь производить вычисления (компьютер справится с этой задачей быстро, точно и без ошибок), но и уметь находить наилучшее решение производственной задачи, характеризуемой экономичностью, простотой, технологичностью и надежностью, а хорошей базой является сопромат.

\section{База знаний для изучения дисциплины}

Пласти́чность - способность материала без разрушения получать большие остаточные деформации. Свойство пластичности имеет решающее значение для таких технологических операций, как штамповка, вытяжка, волочение, изгиб и др.

Пластичностью металлов называется способность металла принимать под действием нагрузки новую форму, не разрушаясь.

Пластичность металлов определяется также при испытании на растяжение. Это свойство обнаруживается в том, что под действием нагрузки образцы разных металлов в различной степени удлиняются, а их поперечное сечение уменьшается. Чем больше способен образец удлиняться, а его поперечное сечение сужаться, тем пластичнее металл образца.

Необходимость определения пластичности металлов вызывается тем, что пластичные металлы можно подвергать обработке давлением, т. е. ковать, штамповать или на прокатных станах превращать слитки металлов в полосы, листы, прутки, рельсы и многие другие изделия и заготовки.

В противоположность пластичным хрупкие металлы под действием нагрузки разрушаются без изменения формы. При испытании хрупкие образцы разрушаются без удлинения, внезапно. Хрупкость является отрицательным свойством. Вполне пригодным для изготовления деталей машин будет не только прочный, но и, в определенной мере, пластичный металл.

Для того чтобы получить представление о пластичности металла и определить величину этого свойства, существуют две единицы измерения: относительное удлинение и относительное сужение при разрыве.

Величина относительного удлинения определяется при испытании следующим образом.

Сначала вычисляется общее удлинение образца при разрыве $1_{1}-1_{0}$, т. е. из его длины в момент разрыва $1_{1}$ вычисляется первоначальная длина $1_{0}$. Полученная разность могла бы служить показателем пластичности металлов только в том случае, если бы длина образцов для испытания была всегда одинаковой.

При различной же начальной длине образцов величина их удлинения для сравнения пластичности металлов является недостаточной, так как длинные образцы будут удлиняться при разрыве больше, чем короткие образцы из того же металла. Поэтому, чтобы иметь возможность сравнивать пластичность различных металлов, необходимо учитывать, какова начальная длина образца и какое он получил удлинение при разрыве относительно первоначальной её длины. Относительное удлинение принято численно выражать в процентах по отношению к первоначальной длине образца и обозначать буквой $\delta$.

Пример. Первоначальная длина образца $1_{0}=200$ мм; длина при разрыве оказалась равной 236 мм; удлинение образца составило $236-200=36$ мм.

Относительное удлинение (\%) при испытании некоторых металлов составляет: для цинка 20, алюминия 40, олова 40 , железа 45 , свинца 45 , никеля 50 , меди 50.

Вторую величину, характеризующую пластичность металлов, - относительное сужение при разрыве $\psi$ - определяют подобным же способом:

$$
\Psi=\frac{F_{0}-F_{1}}{F_{0}} \cdot 100 \%,
$$

где $F_{0}$ - начальная площадь поперечного сечения образца до испытания, мм ${ }^{2} ; F_{1}$ - площадь поперечного сечения образца в месте разрыва, м² $^{2}$.

Таким образом, относительное сужение представляет собой отношение величины уменьшения площади поперечного сечения образца при разрыве к первоначальной площади поперечного сечения. 
Курс дисциплины «Сопротивление материалов» в разделе «Теории предельных состояний» традиционно содержит рассмотрение критерия максимального касательного напряжения Треска Сен-Венана, критерия энергии формоизменения Губера - Мизеса, теории предельных состояний Мора, причем последняя считается самым полным и теоретически обоснованным критерием предельных состояний. При этом используется графоаналитический метод построения круговой диаграммы напряженного состояния, изучение которого не включено в некоторые сокращенные курсы дисциплины «Сопротивления материалов». Можно принять эти условия исключения части материала только при условии, что взамен некоторых установившихся исторически разделов курса создать условия для размышления о задачах, которые обязательно возникнут в практической деятельности инженера. При этом нельзя забывать о том, что появление новых материалов со сложными реологическими свойствами, разработка новых технологий их изготовления методами пластического деформирования ставят ряд принципиально новых задач, решение которых требует глубоких знаний раздела «Теория предельных состояний» курса дисциплины «Сопротивление материалов».

Цель работы: создать и обеспечить порядок изложения теоретического материала раздела «Теории предельных состояний», с опорой на методические приёмы проблемного обучения. При рассмотрении этапов создания проблемной ситуации сформулировать проблему уровня отдельного раздела курса «Сопротивления материалов» при изучении темы «Теория предельных состояний».

\section{Результаты исследования}

Основной задачей инженерных расчётов является оценка прочности конструкции по известному напряжённому состоянию. При незначительных нагрузках материал работает упруго. При больших нагрузках обнаруживаются остаточные деформации, и материал переходит в пластическое состояние. Затем наблюдают накопление повреждений, образование микротрещин, их слияние и наступает состояние разрушения. Под предельным состоянием понимают такое, при котором происходит качественное изменение свойств материала - переход от одного механического состояния к другому. Для пластичных материалов предельнымм обычно считается напряжённое состояние, соответствующее возникновению заметных остаточных деформаций, а для хрупких такое, при котором начинается разрушение.

В связи с этим первый вопрос, который возникает при таком методе расчёта, заключается в определении предельного напряжённого состояния. В случае одноосного напряжённого состояния производится испытание материала на растяжение, и на диаграмме растяжения (впоследствии на диаграмме пластичности $\sigma=f(\varepsilon))$ выбирают характерную точку, соответствующую предельному напряжённому состоянию. Обычно это предел текучести $\sigma_{m}$, либо предел прочности $\sigma_{b}$. Аналогично поступают и в случае чистого сдвига.

Если следовать указанному пути, то для каждого напряжённого состояния, которое характеризуется девятью компонентами тензора напряжений второго ранга в декартовой системе координат, необходимо было бы для каждого материала иметь соответствующие диаграммы испытания с числовыми характеристиками характерных точек $\left(\sigma_{m}\right.$ либо $\left.\sigma_{b}\right)$. В связи с этим возникает проблема: следует ли идти по такому пути, т. е. испытывать ли каждый материал при различных напряжённых состояниях?

На этом этапе необходимо обратить внимание студентов на поставленный вопрос и попытаться получить варианты решений. После анализа решений отвергнуть явно неприемлемые варианты, указав на невозможность испытания каждого материала при различных напряжённых состояниях, прежде всего в силу неисчерпаемости возможных типов напряжённых состояний, а затем и в связи с чисто техническими затруднениями, возникающими при постановке испытаний материалов в условиях плоского и, в особенности, объёмного напряжённого состояния.

В процессе рассмотрения различных предложений возникает необходимость в создании общего метода оценки опасности сложного напряжённого состояния при ограниченном числе механических испытаний материала. Обычно большая часть наших сведений о механических свойствах пластических материалов почерпнута из испытаний на растяжение, в то время как в отношении хрупких материалов они устанавливаются из испытаний на сжатие. Для того, чтобы обосновать назначение допускаемых напряжений в различных случаях сложного напряжённого состояния, в процессе становления науки о сопротивлении материалов выдвигались различные теории прочно- 
сти. Это и является содержанием теории предельных состояний или так называемых теорий прочности.

В настоящее время сложилось два направления в теории предельных состояний. Первое, классическое, направление, связанное с отысканием возможно более точных гипотез, оправданных последующими экспериментами. Второе, исторически более позднее, содержит в своей основе феноменологический подход, т. е. оно основано на выборе наиболее простого и полного описания совокупности экспериментальных данных при минимальных упрощающих предположениях.

Первые классические теории прочности являлись гипотезами о наибольшем главном напряжении, о наибольшей деформации. В соответствии с ними принималось, что текучесть или разрушение при любом сложном напряжённом состоянии начинается, когда наибольшее напряжение или наибольшая деформация достигают определённых критических значений, которые устанавливаются из испытаний на растяжение. Максвелл высказал мысль воспользоваться выражением энергии деформации при оценке прочности в сложном напряжённом состоянии. Он утверждал: «Я первый берусь писать на эту тему. Мне ещё не приходилось видеть ни одной научной работы, в которой при заданных трёх направлениях механических воздействий на элемент решался бы вопрос об его несущей способности». Мы видим, что Максвелл владел теорией прочности, которую теперь называют теорией энергетического формоизменения. Но он никогда больше не возвращался к этому вопросу, и его мысль стала известной только после издания его писем. Энергетическая теория прочности основана на гипотезе, что напряжённые состояния равноопасны в том случае, если у них совпадает величина удельной потенциальной энергии формоизменения (критерий пластичности Мизеса).

Как возникали эти гипотезы? Известно, что при деформировании возникают нормальные и касательные напряжения, деформации, накапливается потенциальная энергия. Поэтому в качестве критерия естественным кажется принятие одного из перечисленных факторов: наибольших нормальных, касательных напряжений, наибольших линейных деформаций, удельной потенциальной энергии формоизменения. При этом следует привлечь внимание студентов и попытаться получить ответ на вопрос: какими могут быть гипотезы, исходя из ограничений по напряжениям? Аналогичная ситуация проигрывается для случаев ограничения деформации или потенциальной энергии формоизменения. После принятия оптимальной гипотезы указывается общий метод создания теории прочности, излагается его сущность.

При одноосном напряжённом состоянии граница прочности оценивается предельным (опасным) значением напряжения. При переходе к сложному напряжённому состоянию естественно ввести простейшее обобщение этой гипотезы - предельную поверхность в пространстве напряжений. Для изотропного тела все направления эквивалентны, поэтому уравнение этой поверхности должно быть инвариантным по отношению к ориентации координатной системы, следовательно, оно должно содержать лишь инварианты напряжённого состояния, т. е. иметь вид:

$$
F\left[J_{1}\left(T_{\sigma}\right), J_{2}\left(T_{\sigma}\right), J_{3}\left(T_{\sigma}\right)\right]=K \text {. }
$$

Можно рассматривать поверхность в пространстве главных напряжений

$$
F\left[\sigma_{1}, \sigma_{2}, \sigma_{3}\right]=K .
$$

При линейном растяжении условием для перехода от упругого состояния к пластическому является равенство:

$$
\sigma_{1}=\sigma_{m}
$$

Условия перехода материала в пластическое состояние при любом виде напряжённого состояния и составляют суть гипотез прочности.

Гипотезы, определяющие условия перехода напряжённого тела от упругого состояния к пластическому, сокращенно называют «условиями пластичности». Энергетическая теория в принятой в настоящее время формулировке была предложена М. Губером (1914) и Ричардом фон Мизесом (1913). Как и теория наибольших касательных напряжений, энергетическая теория, по сути, устанавливает критерий появления в конструкции необратимых деформаций, причём результаты расчётов по этим теориям довольно близки и хорошо согласуются с экспериментами на образцах из пластичных материалов. Это условие можно сформулировать нижеизложенным образом. 
Любая элементарная частица металлического тела переходит из упругого состояния в пластическое, когда интенсивность напряжений достигает значения, равного напряжению текучести при линейном пластическом напряжённом состоянии.

Иначе говоря, при пластическом состоянии интенсивность напряжений постоянно равна напряжению текучести:

$$
\sigma_{u}=\frac{1}{\sqrt{3}} \sqrt{\left(\sigma_{1}-\sigma_{2}\right)^{2}+\left(\sigma_{2}-\sigma_{3}\right)^{2}+\left(\sigma_{3}-\sigma_{1}\right)^{2}}=\sigma_{m} .
$$

Если в условии пластичности (3), рассматривать $\sigma 1, \sigma 2, \sigma 3$ как текущие координаты, то уравнение примет вид:

$$
\frac{1}{\sqrt{3}} \sqrt{\left(\sigma_{1}-\sigma_{2}\right)^{2}+\left(\sigma_{2}-\sigma_{3}\right)^{2}+\left(\sigma_{3}-\sigma_{1}\right)^{2}}=2 \sigma_{m}^{2}
$$

Выражение (4) представляет собой поверхность цилиндра радиусом $r$, неограниченного по длине образующей цилиндра, ось которого проходит через начало координат и одинаково наклонена к осям координат $\sigma_{1}, \sigma_{2}, \sigma_{3}$, т. е. составляет с каждой из них угол, косинус которого равен $\frac{1}{\sqrt{3}}$.

Если главные нормальные напряжения в каком-либо элементе твёрдого тела таковы, что они определяют точку, лежащую на поверхности цилиндра, этот элемент будет в пластическом состоянии. Таким образом, поверхность, согласно уравнению (1), является «предельной поверхностью пластической деформации» согласно энергетическому условию пластичности. Графически этот цилиндр представлен на рис. 1.

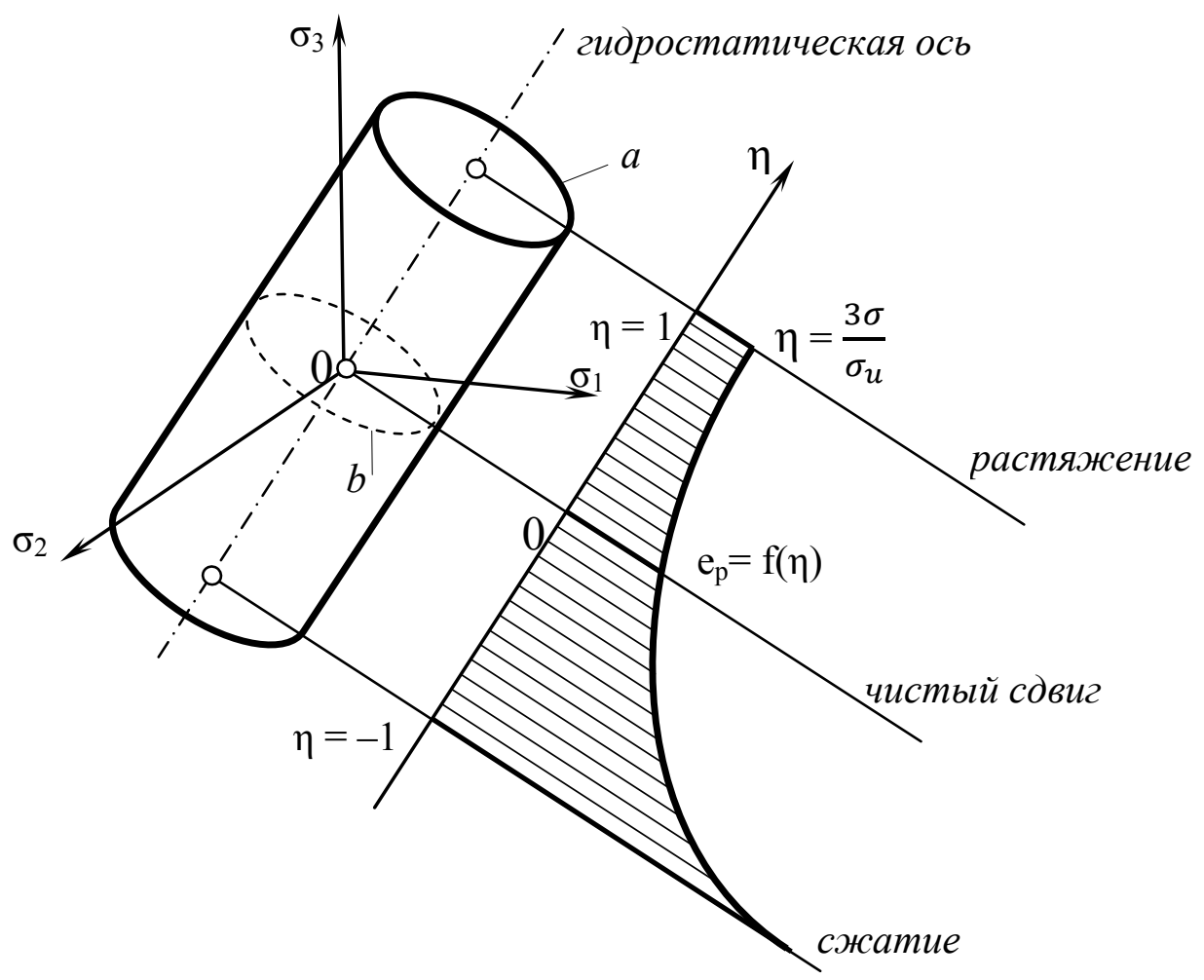

Рисунок 1 - Предельная поверхность напряжений согласно энергетическому условию пластичности

Если главные нормальные напряжения в элементе тела таковы, что они определяют точку, лежащую внутри цилиндра, то элемент будет находиться в упругом напряжённом состоянии. Комбинация напряжений и определяющие точки вне поверхности цилиндра не имеют физического смысла. Чем больше напряжение текучести $\sigma_{m}$, тем больше и радиус цилиндра $r$. Если деформация сопровождается упрочнением, $\sigma_{m}$ увеличивается и, следовательно, предельная поверхность пластической деформации расширяется.

Окружности на поверхности цилиндра (например, $a, b$ ), полученные пересечением его плоскостями, перпендикулярными к его оси, представляют собой геометрическое место точек, опреде- 
ляющих предельные напряжённые состояния, т. е. напряжённые состояния с одинаковым шаровым тензором (гидростатическим давлением), для которых сумма главных нормальных напряжений - постоянная величина. Это вытекает из того, что уравнением плоскостей, нормальных к оси цилиндра, является уравнение:

$$
\sigma_{1}+\sigma_{2}+\sigma_{3}=P \sqrt{3}
$$

где $\mathrm{P}$ - длина нормали, проведённой из начала координат к этой плоскости.

В частности, для круга $b$, образованного пересечением цилиндра плоскостью, проходящей через начало координат 0, эта сумма равна нулю (т. е. напряжённое состояние является чисто девиаторным).

Заметим, что при гидростатическом сжатии однородный материал не разрушается, а начало текучести, согласно (3), не зависит от уровня гидростатического давления. Поэтому ось цилиндра, показанная на рис. 1, проходит через начало координат и характеризуется одинаковыми направляющими косинусами $\frac{1}{\sqrt{3}}$. Движение по гидростатической оси (это означает изменение гидростатического давления) не вызывает изменения $\sigma_{m}$ (радиус цилиндра остается постоянным).

Таким образом, конкретизация зависимости (2) приводит к тем или иным критериям прочности. Здесь, как уже подчёркивалось, в качестве критерия разрушения использовались ограничения, накладываемые на напряжения. С помощью условия (3), зная главные напряжения, можно достоверно предсказать наступление предельного состояния, выражающего переход от упругого состояния к пластическому.

Ещё раз следует обратить внимание на то обстоятельство, что критерий (3) основан на ограничениях, накладываемых на напряжения. Наряду с рассмотренным условием текучести излагаются $I$, $I I$, III классические теории прочности, указываются их достоинства и недостатки и, естественно, возникает вопрос: каково предельное состояние за пределами упругости? На рис 2 показана кривая течения малоуглеродистой стали, построенная на данных испытаний образцов на двухосное растяжение, сжатие, одноосное растяжение и кручение. В теории пластичности доказано, что в координатах интенсивность напряжений $\sigma_{u}$ и интенсивность деформаций $e_{u}$ связаны соотношением:

$$
\sigma_{u}=\sqrt{\left(\mathrm{e}_{1}-\mathrm{e}_{2}\right)^{2}+\left(\mathrm{e}_{2}-\mathrm{e}_{3}\right)^{2}+\left(\mathrm{e}_{3}-\mathrm{e}_{1}\right)^{2}} .
$$

Кривая $\sigma_{u}=f\left(e_{u}\right)$ является единой и независимой от вида напряжённого состояния. При построении указанной кривой путем испытания образцов на растяжение, кручение и сжатие поступают следующим образом. При растяжении $\sigma_{u}=\frac{4 P}{\pi d_{0}^{2}} \frac{h_{i}}{h_{0}}, e_{u}=2 \ln \frac{d_{0}}{d_{i}} ;$ следовательно, в процессе растяжения измеряют диаметр образца на промежуточных стадиях вплоть до разрушения. На рис. 2 показаны точки $1,2,3,4,5$, отвечающие значениям $\sigma_{u}$ и $e_{u}$ в момент разрушения в условиях двухосного растяжения, растяжения, чистого сдвига и сжатия.

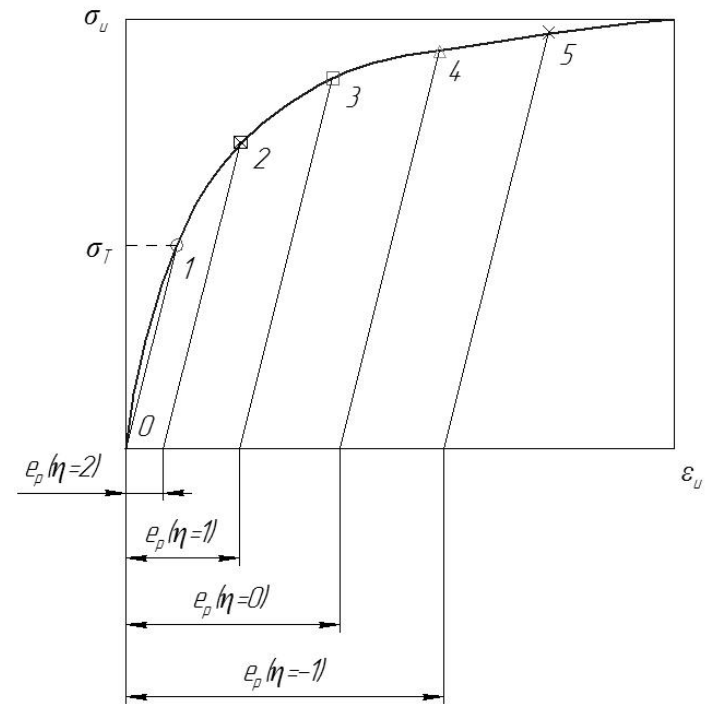

Рисунок 2 - Вид кривой течения малоуглеродистой стали

При кручении интенсивность напряжений 


$$
\sigma_{u}=\frac{\sqrt{3}}{2 \pi r^{3}}\left(3 M+\gamma \frac{d M}{d \gamma}\right)
$$

где $M$ - крутящий момент; $\gamma$ - сдвиг на поверхности закручиваемого цилиндрического образца

$$
\gamma=\operatorname{tg} \alpha=\frac{\varphi d}{2 l}
$$

где $\varphi$ - угол закручивания; $l$ - рабочая длина; $d$ - диаметр образца, $\alpha$ - угол сдвига на поверхности.

Угол сдвига $\alpha$ измеряют на инструментальном микроскопе между продольной осью образца и чертой, проведенной до испытания вдоль образующей оси. В момент разрушения предельная ин-

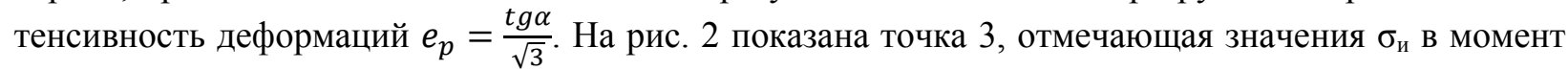
разрушения при кручении. Кривая строится испытанием образцов на сжатие следующим образом. Из исследуемого материала изготавливают цилиндрические образцы $\frac{h_{0}}{d_{0}}=1,5\left(\mathrm{~d}_{0}=10 \ldots 20\right.$ мм). Затем их осаживают в условиях смазки контактных поверхностей до различных степеней деформации вплоть до разрушения (появления видимой макротрещины). Интенсивность напряжений и деформаций на каждой стадии деформирования рассчитывают по формулам:

$$
\sigma_{u}=\frac{4 P}{\pi d_{0}^{2}} \frac{h_{i}}{h_{0}} ; \quad e_{u}=\ln \frac{h_{0}}{h_{i}},
$$

где $h_{0}, h_{i}$ - высоты образцов до и после осаживания.

На рис. 2 точкой 5 отмечено значение предельной деформации и интенсивности напряжений при сжатии в момент разрушения. Как следует из рис. 2 , значение $\sigma_{u}$, полученное в условиях различных видов напряжённого состояния (растяжение, сжатие, кручение), отличается незначительно (отличие зависит от способности материала к упрочнения). Для идеально пластичных материалов $\sigma_{u}=$ const. Однако значения предельных интенсивностей деформации при различных видах деформации отличаются существенно.

Следовательно, здесь удаётся столкнуть студента с фактом непригодности условия (3) для оценки предельного состояния за пределами упругости.

Таким образом, для пластичных материалов за пределами упругости предельное состояние зависит (см. рис. 1) от относительного гидростатического давления. В качестве меры предельного состояния в рассматриваемом случае не может быть принято напряжение текучести. Почему? На этот вопрос следует обратить внимание студентов и попытаться получить варианты ответов.

В качестве меры предельного состояния в рассматриваемом случае следует принять предельную интенсивность деформации, накопленную в опасной частице материала вплоть до разрушения. При различных видах напряжённого состояния необходимо уметь определять интенсивность деформации к моменту разрушения (в дальнейшем будем называть её предельной деформацией $\left.e_{p}\right)$. Она и является мерой пластичности материалов. Как было показано на рис. 1, значение предельной деформации оказалось различным при растяжении, кручении (сдвиге) и сжатии. Повидимому, пластичность материалов нельзя во всех случаях характеризовать относительным остаточным сужением $\left(\psi=\frac{F_{0}-F_{i}}{F_{0}}\right)$ или относительным остаточным удлинением $\left(\delta=\frac{l_{i}-l_{0}}{l_{0}}\right)$, так как эти механические характеристики пластичности отражают способность материала выявить пластические деформации лишь при одном виде испытания, например, при одноосном растяжении. В общем же случае пластического деформирования пластичность существенно зависит от схемы напряжённого состояния, характеризуемой относительным гидростатическим давлением

$$
\eta=\frac{\sigma_{1}+\sigma_{2}+\sigma_{3}}{\sigma_{u}}=\frac{3 \sigma}{\sigma_{u}}
$$

где $\sigma_{1}, \sigma_{2}, \sigma_{3}$ - главные напряжения; $\sigma_{u}-$ интенсивность напряжений, $\sigma-$ среднее напряжение (гидростатическое давление).

Показатель $\eta$ отражает влияние относительного гидростатического давления и является безразмерной величиной. Показатель $\eta$ - величина инвариантная (по отношению к выбору координатных осей), но не лишенная физического смысла. Действительно, пластичность зависит от среднего напряжения; с увеличением всестороннего давления пластичность растет, хрупкие материалы в таком случае могут деформироваться. Однако один лишь гидростатический фон без собственно деформации (растяжения, сдвига и т. д.) не вызывает формоизменения. 
Пластическая деформация возникает тогда, когда интенсивность напряжений $\sigma_{u}$ достигает напряжения текучести $\sigma_{m}(3)$, поэтому среднее напряжение относят к интенсивности напряжений.

Зависимость предельной деформации от показателя $\eta$ названа диаграммой пластичности $e_{p}=f(\eta)$. Строят её испытанием материала на сжатие, кручение и растяжение, иногда на двухосное растяжение.

Показатель $\eta=1$ при растяжении

$$
\eta=\frac{\sigma_{1}+\sigma_{2}+\sigma_{3}}{\sigma_{u}}=\frac{\sigma_{u}}{\sigma_{u}}=1
$$

При сжатии

$$
\eta=-\frac{\sigma_{1}}{\sigma_{u}}=-1
$$

При чистом сдвиге (кручении)

$$
\eta=\frac{-\tau+0-\tau}{\sigma_{u}}=0
$$

При двухосном растяжении

$$
\eta=\frac{\sigma_{1}+\sigma_{2}+\sigma_{3}}{\sigma_{u}}=\frac{\sigma_{1}+\sigma_{2}+0}{\sigma_{u}=\sigma_{1}}=2 .
$$

Предельные деформации при этих значениях показателя:

$$
\begin{gathered}
\sigma_{u}=f(\eta=1)=2 \ln \frac{d_{0}}{d_{i}=d_{\amalg}}, \\
\sigma_{u}=f(\eta=0)=\frac{\operatorname{tg} \alpha}{\sqrt{3}}, \\
\sigma_{u}=f(\eta=2)=2 \ln \frac{d}{d_{0}},
\end{gathered}
$$

где $d_{0}, d_{ш}, d$ - диаметры образцов в исходный момент и в момент разрушения.

Таким образом, диаграммы пластичности $e_{p}=f(\eta)$ расширяют наши представления о механических характеристиках материалов. В. Огородниковым (1977) предложены различные аппроксимации диаграмм пластичности, с помощью которых удаётся, не проводя трудоёмких испытаний, рассчитать $e_{p}$ (оценить пластичность) при любом значении показателя $\eta$.

Так, для материалов, у которых

$$
\lambda_{1}=\frac{e_{p}(\eta=-1)}{e_{p}(\eta=0)}<\frac{e}{2}
$$

где $e \approx 2,72$;

аппроксимирующее уравнение имеет вид

$$
e_{p}(\eta)=e_{p}(\eta=0) \exp \left[\left(2-\lambda_{1}\right)-\eta\right]
$$

Уравнение (14) описывает диаграмму в области изменения показателя $1 \leq \eta \leq 3$.

Для материалов, у которых $\lambda_{2}=\frac{e_{p}(\eta=0)}{e_{p}(\eta=1)} \leq e$, уравнение, описывающее диаграммы пластичности, имеет вид $e_{p}(\eta)=e_{p}(\eta=0) \exp \left[\left(-\ln \lambda_{1}\right) \eta\right]$.

Введение в расчётную практику диаграмм пластичности в координатах $e_{p}=f(\eta)$ позволяет решить ряд важных практических задач, направленных на внедрение прогрессивных технологий, а также на оптимизацию расчётов стальных конструкций по критериям предельных пластических деформаций. Технологические процессы обработки металлов давлением (прокатка, ковка, штамповка, волочение, прессование, дорнование, ротационная ковка и др.) основаны на способности металла пластически деформироваться. Расчёты таких процессов необходимы для установления энергосиловых параметров, установления параметров, обеспечивающих высокое качество изделий. В последнее время наряду с указанными параметрами особое значение приобретают методы оценки деформируемости материалов без разрушения, позволяющие на стадии проектирования 
технологических процессов способствовать его интенсификации и предотвращению разрушения материала в процессе формоизменения. Имеющиеся теории прочности, о которых уже говорилось, основаны, главным образом, на ограничениях, накладываемых на напряжения. В этих критериях подразумевается, по существу, простое нагружение. В более общем случае в области больших пластических деформаций реализуется сложное нагружение, поэтому фиксированной предельной поверхности не будет, так как условие разрушения зависит от истории деформирования. Именно поэтому следует искать не вид предельной поверхности (её математическое описание), а формулировать условие деформируемости без разрушения, учитывающего влияние истории деформирования. При этом условие или критерий деформируемости, естественно, должен быть основан на ограничениях, накладываемых на деформации (ограничения на напряжения, в частности, на эквивалентное напряжение не приведет к разумным результатам, так как в случае $\sigma_{u}=$ const деформации могут увеличиваться сколько угодно, а разрушение может не наступить).

Один из первых критериев деформируемости металлов без разрушения был предложен Г. А. Смирновым-Аляевым (1968). Он имеет следующий вид

$$
e_{u}=\int \dot{\varepsilon}_{u} d \tau \leq e_{p}(\eta)
$$

или, нормируя на единицу в момент разрушения,

$$
\psi=\frac{e_{u}}{e_{p}(\eta)} \leq 1,
$$

где $e_{p}(\eta)$ - предельная деформация в момент появления первых трещин, обнаруживаемых визуально; $\psi$ - использованный ресурс пластичности, который при деформировании без разрушения меньше единицы.

В критерии (15) величина $e_{p}(\eta)$ отражает зависимость пластичности от показателя напряжённого состояния (диаграмма пластичности). При оценке величины $\psi$ по Г. А. Смирнову-Аляеву влиянием истории деформирования (изменением показателя $\eta$ с ростом степени деформации $e_{u}$ ) пренебрегают; предельную деформацию, при которой происходит разрушение, определяют лишь по показателю $\eta$.

В. Л. Колмогоров (1970) предложил следующий критерий деформируемости без разрушения:

$$
\psi=\int_{0}^{e_{u}^{\prime}} B \frac{d e_{u}}{e_{p}\left(e_{u}\right)} \leq 1 .
$$

Здесь коэффициент $B$ призван учитывать влияние истории деформирования. Однако значение $B$ для различных историй деформирования остается невыясненным. Вследствие чего его часто принимают равным единице. Критерий (16) исходит из линейной теории накопления повреждений, которая в общем случае деформирования даёт систематические отклонения расчётных и экспериментальных данных. Критерий деформируемости, учитывающий влияние истории деформирования и основанный на нелинейной теории накопления повреждений, предложен в работе (Огородников, 1977). Он записывается в виде:

$$
\psi=\int_{0}^{e_{u}^{\prime}}\left(1+0,2 \operatorname{arctg} \frac{d \eta}{d e_{u}}\right) \frac{e_{u}^{0,2 \operatorname{arctg} \frac{d \eta}{d e_{u}}}}{\left[e_{p\left(e_{u}\right)}\right]^{1+0,2 \operatorname{arctg} \frac{d \eta}{d e_{u}}}} \leq 1 .
$$

Здесь $\frac{d \eta}{d e_{u}}$ - параметр, учитывающий влияние истории деформирования на пластичность. Для того, чтобы воспользоваться критерием (17), необходимо путём простейших испытаний (растяжением, кручением, сжатием) выявить вид функции $e_{p}=f(\eta)$, а затем - изменение показателя $\eta$ с ростом интенсивности деформации в опасной точке очага деформации в процессах формоизменения.

Применение теории деформируемости металлов без разрушения (Сторожев \& Попов, 1971), развитой в работах С. И. Губкина, Г. А. Смирнова-Аляева, В. Л. Колмогорова, В. А. Огородникова, изложено в работах Смирнова-Аляева (1968), Огородникова (1977). В результате применения усовершенствованной теории удалось интенсифицировать многие технологические операции обработки металлов давлением, с точки зрения повышения деформируемости заготовок, и получить существенный экономический эффект, связанный с уменьшением брака от разрушения материалов. 
Однако создание теорий предельного состояния по-прежнему остается актуальной задачей. Так, несмотря на то, что условие деформируемости (17) позволяет оценивать предельное состояние с учётом истории деформирования, оно не позволяет рассчитывать предельные деформации в процессах, сопровождающихся циклическим деформированием. Опыты показывают, что на пластичность оказывает влияние также третий (кубический) инвариант тензора напряжений $\left(J_{3}\left(T_{\sigma}\right)=\sigma_{1} \sigma_{2} \sigma_{3}\right)$, что не отражено в критерии (16).

\section{Выводы}

Проанализированы содержание и порядок изложения теоретического материала раздела «Теории предельных состояний» с опорой на методические приемы проблемного обучения. При рассмотрении этапов создания проблемной ситуации сформулирована проблема уровня отдельного раздела курса «Сопротивления материалов» при изучении темы «Теория предельных состояний».

Таким образом, развитие дисциплин «Сопротивление материалов», «Механика материалов и конструкций», а также требования практики оказывают влияние на дальнейшее развитие теории предельных состояний.

\section{СПИСОК ПОСИЛАНЬ}

Колмогоров, В. Л. (1970). Напряжения. Деформации. Разрушение. Москва: Металлургия.

Огородников, В. А. (1977). Оценка деформируемости металлов при обработке давлением. Кузнечно-итамповочное производство, 3, 15-18.

Смирнов-Аляев, Г. А. (1968). Механические основы пластической обработки металлов. Ленинград: Машиностроение.

Сторожев, М. В., \& Попов, Е. А. (1971). Теория обработки металлов давлением. Москва: Машиностроение.

Феодосьев, В. И. (2010). Сопротивление материалов. Москва: МГТУ им. Баумана.

Dell, Y., Gese, H., Kessler, L., Werner, H., \& Hooputra, H. (2001). Continuous Failure Prediction Model for Nonlinear Load Paths in Successive Stamping and Crash Processes. World Congress, Detroit, Michigan, March 5-8, 2001. New Sheet Steel Products ans Sheet Metal Stamping. SAE Proceeding SP-1614, paper 2001-01-1131, 113-122.

\section{REFERENCES}

Dell, Y., Gese, H., Kessler, L., Werner, H., \& Hooputra, H. (2001). Continuous Failure Prediction Model for Nonlinear Load Paths in Successive Stamping and Crash Processes. World Congress, Detroit, Michigan, March 5-8, 2001. New Sheet Steel Products ans Sheet Metal Stamping. SAE Proceeding SP-1614, paper 2001-01-1131, 113-122.

Feodosev, V. I. (2010). Soprotivlenie materialov. Moskva: MGTU im. Baumana.

Kolmogorov, V. L. (1970). Napryazheniya. Deformatsii. Razrushenie. Moskva: Metallurgiya.

Ogorodnikov, V. A. (1977). Otsenka deformiruemosti metallov pri obrabotke davleniem. Kuznechnoshtampovochnoe proizvodstvo, 3, 15-18.

Smirnov-Alyaev, G. A. (1968). Mehanicheskie osnovyi plasticheskoy obrabotki metallov. Leningrad: Mashinostroenie.

Storozhev, M. V., \& Popov, E.A. (1971). Teoriya obrabotki metallov davleniem. Moskva: Mashinostroenie.

\section{Віталій Огородніков}

Тетяна Архипова

Андрій Губанов

\section{Елементи проблемного навчання теорії граничних станів в задачах опору матеріалів}

Виконано аналіз змісту та порядку викладання теоретичного матеріалу розділу «Теорії граничних станів» 3 опорою на методичні прийоми проблемного навчання. При розгляді етапів створення проблемної ситуації сформульована проблема рівня окремого розділу курсу «Опір матеріалів». Розкриті особливості історичного розвитку поняття «граничний стан» дисциплін «Опір матеріа- 
лів», «Механіка матеріалів і конструкцій». Описано структуру лекції, сформульовано проблемні ситуації, отримано розв'язання проблем розвитку основних понять. Підкреслено необхідність врахування вимог практики, яка впливає на подальший розвиток теорії граничних станів.

Ключові слова: теорія граничних станів, принципи проблемного навчання, професійна підготовка інженерів-механіків, критерії міцності.

Вімалій Огородніков - доктор технічних наук, професор, завідувач кафедри опору матеріалів та прикладної механіки, Вінницький національний технічний університет, Вінниця, e-mail: va.ogorodnikow@gmail.com

Tетяна Apxinoва - кандидат технічних наук, доцент, доцент кафедри опору матеріалів та прикладної механіки, Вінницький національний технічний університет, Вінниця, e-mail: tfarhipova@gmail.com

Андрій Губанов - завідувач лабораторіями кафедри опору матеріалів та прикладної механіки, Вінницький національний технічний університет, Вінниця, e-mail: gubanovav@ukr.net

\section{Ohorodnikov}

\section{T. Arkhipova}

\section{A. Gubanov}

\section{Elements of problem training theory of limit states in the problems of strength of materials}

Competent-professional approach in higher education involves a special organization of the pedagogical process of institution of higher education. The practical, interdisciplinary, applied aspects of education are being strengthened. This is achieved not due to the introduction of new subjects or an increase in the volume of disciplines, but due to their substantive reorientation from "declarative" knowledge (to know "what") to procedural (to know "why and why").

While the existing qualification approach is aimed at developing a graduate system of knowledge and skills that provide typical professional activities in stable conditions, the competence approach contributes to the formation of readiness for the optimal solution of professional, social, personal problems in rapidly changing circumstances of the information society. Modern approaches require educational processes of studying such classical disciplines as chemistry, physics, resistance of materials and others.

The content and order of presentation of the theoretical material of the section "Theory of limit states" are analyzed with the support of methodological methods of problem training. When considering the stages of creating a problem situation, the problem of the level of a separate section of the course "Strength of materials" is formulated. The features of historical development of the concept of the "limiting state" of disciplines are revealed. The structure of the lecture is described, problem situations are formulated, solutions are obtained for the development of basic concepts. The need to take into account the requirements of practice that influence the further development of the theory of limit states is underlined.

Keywords: theory of limit states, principles of problem training, vocational training of a mechanical engineer, strength criteria.

Ohorodnikov Vitalii - Dr. Sc. (Eng.), Professor, Head of the Chair of Strength of Materials and Applied Mechanics, Vinnytsia National Technical University, Vinnytsia, e-mail: va.ogorodnikow@gmail.com

Arkhipova Tatiana - Cand. Sc. (Eng.), Assistant Professor, Assistant Professor of the Chair of Strength of Materials and Applied Mechanics, Vinnytsia National Technical University, Vinnytsia, e-mail: tfarhipova@gmail.com

Gubanov Andrii - Head of Laboratories of the Chair of Resistance of Materials and Applied Mechanics, Vinnytsia National Technical University, Vinnytsia, e-mail: gubanovav@ukr.net 
Виталий Огородников

Татьяна Архипова

Андрей Губанов

\section{Элементы проблемного обучения теории предельных состояний в задачах сопротивления материалов}

Проанализированы содержание и порядок изложения теоретического материала раздела «Теории предельных состояний» с опорой на методические приёмы проблемного обучения. При рассмотрении этапов создания проблемной ситуации сформулирована проблема уровня отдельного раздела курса «Сопротивления материалов». Раскрыты особенности исторического развития понятия «предельное состояние» дисциплин «Сопротивление материалов», «Механика материалов и конструкций». Описана структура лекции, сформулированы проблемных ситуации, получены решения проблем развития основных понятий. Подчеркнута необходимость учёта требований практики, оказывающих влияние на дальнейшее развитие теории предельных состояний.

Ключевые слова: теория предельных состояний, принципы проблемного обучения, профессиональная подготовка инженера-механика, критерии прочности. 